\title{
Clinical and functional outcome in a subject with bipolar disorder and severe white matter hyperintensities
}

\author{
Gianluca Serafini ${ }^{\star}$ \\ Maurizio Pompili*,** \\ Massimiliano Angelone*

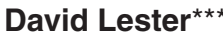 \\ Paolo Girardi* \\ Roberto Tatarelli* \\ * Department of Psychiatry - Sant'Andrea \\ Hospital, "Sapienza" University of Rome \\ ** McLean Hospital - Harvard Medical \\ School, Boston \\ *** The Richard Stockton College \\ of New Jersey \\ ITALY \\ USA
}

\begin{abstract}
Background and Objectives: Neuroimaging studies have found higher rates of white matter hyperintensities (WMHs) in patients with bipolar disorder (BD) of all ages, although whether BD is associated with increased rates of WMHs independently from age and cerebrovascular risk factors is still matter of debate. The outcome of BD associated with severe WMHs is generally poor, but several authors have suggested that some factors could have a protective role in BD. The aim of the present study was to report the two-year follow-up of a woman with BD type I and severe WMH/PWMH lesions who was taking high concentrations of vitamin-D in her nutrition, as well as taking lithium and haloperidol as treatment.

Case presentation: A 76-year-old woman was hospitalized for a mixed state BD. She had severe WMHs. She took lithium and haloperidol during the hospitalization and was euthymic at discharge as well as after two-years of follow-up. Her nutrition had a high concentration of Vitamin-D. Unfortunately, it was not possible to give her a second MRI.

Conclusions: Although there was probable persistence of WMHs, the patient improved in both mood and quality of life. The possible protective effect of lithium and Vitamin-D is discussed.
\end{abstract}




\section{Background and objectives}

Neuroimaging studies have found higher rates of WMHs in patients of all ages with bipolar disorder (BD), most frequently localized in the frontal lobes and the frontal/parietal junction ${ }^{1}$. WMHs may indicate astrogliosis, demyelination and loss of axons and may be relatively more common in older patients with $\mathrm{BD}$, reflecting an interaction of the disease with processes of normal aging. However, WMHs are also associated with several pathological conditions among older individuals ${ }^{2}$. As a result of this, the meaning of these lesions in BD is still unclear.

Although there have been inconsistent results in the research on this issue, WMHs are considered to be negative prognostic factors, associated with treatment resistance, increased hospitalization rates, cognitive impairment and increased suicide risk in individuals with $\mathrm{BD}^{3,4}$.

However, several other factors may play a protective role in $\mathrm{BD}$. Tsai et al. ${ }^{5}$ reported that psychiatric treatment, including medication with antipsychotics or lithium, could be a protective factor against early natural death. Here, we present the case of a 76year-old woman who had had a BD for twenty-one years and had, in addition, severe WMH/PWMH lesions, who was admitted to our psychiatric hospital for a mixed state. The patient gave written consent before being included in the study.

\section{Case presentation}

A 76-year-old woman was admitted to our psychiatric hospital in a mixed state. She had elevated mood, social withdrawal, weight loss and inner tension, and she was agitated and dysphoric. She also presented with delusional persecutory ideas and auditory hallucinations.

She had been diagnosed as having BD type I at the age of 55, but she had never been hospitalized. She had ten previous mood episodes ( 5 depressive, 3 manic and 2 mixed episodes), but she had no actual comorbid psychiatric condition.

She had no brain injuries at birth, data confirmed by her sister during the psychiatric interview. She had no history of hypertension, diabetes, or heart failure. Her cholesterol level was $150 \mathrm{mg} / \mathrm{dl}$. She had normal blood pressure values during the hospitalization (as confirmed by the Holter electrocardiogram). She took lithium at $150 \mathrm{mg} / \mathrm{die}$ from the illness onset, but recently this had been stopped without any specific reason given. A brain magnetic resonance imaging scan revealed severe confluent periventricular WMHs (see Fig. 1). The presence of WMHs was assessed by a neuroradiologist blind to all clinical information, using the modified Fazekas rating scale ${ }^{6}$.

While hospitalized, she was treated with haloperidol $2 \mathrm{mg}$ and lithium carbonate 300 mg daily. She improved rapidly and was discharged after two week of hospitalization. At discharge, her Young mania rating scale score (YMRS $)^{7}$ had decreased from 30 to 12 and the Hamilton depression rating scale score (HDRS $)^{8}$ from 26 to 16 . The physical component (PC) score of the Short Form Health Survey (SF-36) ${ }^{9}$ had increased from 10 to 22 and mental component (MC) score from 10 to 18. She was euthymic for the following two-years of follow-up. Psychometric measures were repeated every year and remained stable.

She was a vegetarian and frequently ate fat fish, cod-liver oil, eggs and milk which have high concentrations of vitamin-D. Se- 


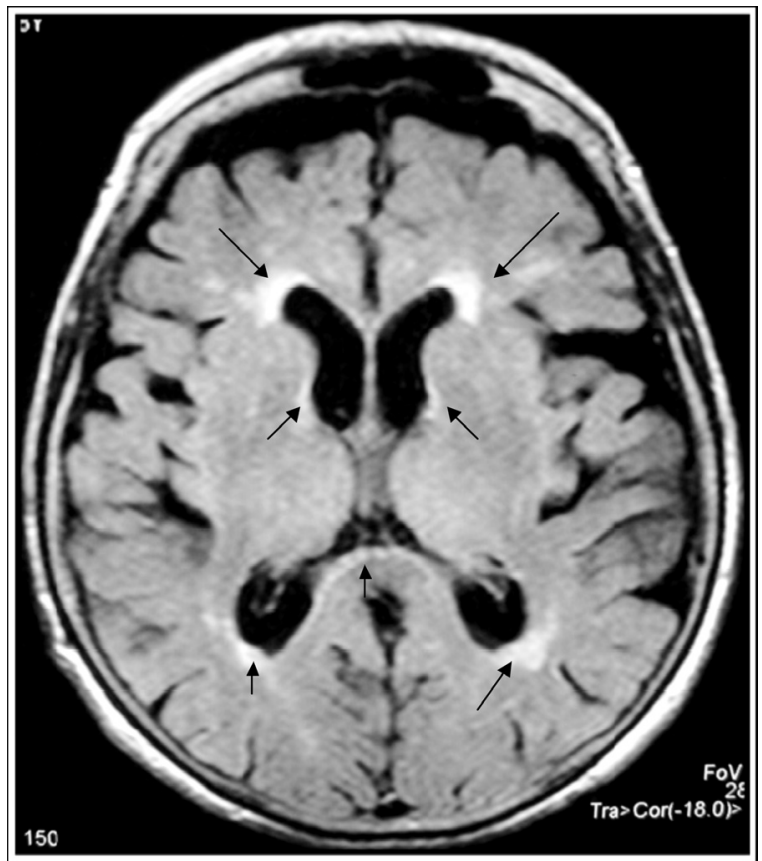

Figure 1. Axial T2-weighted (PD and T2 ax: TR 2870; TE 13/107; thickness 5 mm; matrix 147_256) in the axial and the coronal planes) brain magnetic resonance imaging performed using a Siemens Sonata, Erlangen, Germany (1.5 T). The FLAIR scan sequence was used for WMH measurement (ax: TR 10000; TE 125; thickness 5 mm; matrix 144_256).

The Fazekas four-point rating scale describes MRI hyperintensities on an ascending scale of intensity and frequency. The Fazekas scale indicated in this case, as indicated by the arrows the presence of large confluent PWMHs and WMH lesions ( 3 and 3 were respectively observed as scores of severity).

rum lithium levels were within the range (0.6-1.2 meq/L) and were monitored every month. Her Mini Mental State Examination score (MMS) ${ }^{10}$ increased from 22 to 26 and remained stable during the follow-up period. Unfortunately, it was not possible to administer a second MRI because she was surgically implanted with a left hip prosthesis for coxarthritis.

\section{Discussion}

The most relevant finding of this case report is that, despite the presence of severe WMHs, our patient was euthymic during the two-years of follow-up and had good psychosocial functioning.

In most patients with $\mathrm{BD}$, there is a gap between clinical remission and functional outcome $\mathrm{e}^{11,12}$ and, after a relapse, only $40 \%$, although euthymic, achieve their premorbid functional status ${ }^{13}$. Therefore, it is important to identify which factors may contribute to this disability. Recently, Rosa et al. ${ }^{14}$ suggested that the poor functional outcome in patients with $\mathrm{BD}$ was predicted by the previous number of mixed episodes, current subdepressive symptoms, the previous number of hospitalizations and an older age. Our patient had no previous mixed episodes, no current subdepressive symptoms, no previous hospitalizations and a later age of onset 
of bipolar illness, but, interestingly, she also had a nutrition rich in vitamin-D which is a multipurpose steroid hormone vital to health and increasingly implicated in the pathology of cognition and mental illness. The vitamin-D receptors and the vitamin-D activating enzyme 1-alpha-hydroxylase are widely distributed in the human brain ${ }^{15}$. Hypovitaminosis D may be common among older adults, and it is associated with poor executive performance in patients with mood disorders ${ }^{16}$. Vitamin-D receptors activate neurons implicated in the regulation of behaviour, stimulate neurotrophin release and protect the brain, presumably by buffering antioxidant and anti-inflammatory defenses against vascular injury ${ }^{17}$. Langub et al. ${ }^{18}$ reported that vitamin-D plays a neuroprotective role (e.g., an anti-oxidative effect) for hippocampal cell survival, presumably mitigating cellular homeostasis. Although poorly understood, the eventual neuroprotective effect of vitamin-D in subjects with mood disorders may be mediated by their antioxidative and/or anti-inflammatory action.

Our patient took lithium which may promote neurogenesis both in short and long term treatment ${ }^{19}$. Lithium treatment has demonstrated a robust neuroprotective role in preventing apoptosis of neurons, and this has relevant clinical implications ${ }^{20,21}$. Although no direct evidence of lithium's ability to improve cognitive performance has been found, lithium-induced increases in gray matter volume may be associated with a positive clinical response, particularly during weeks 10-12 and maintained through 16 weeks of treatment ${ }^{22}$.

\section{Conclusions}

Although limited to the present case report, our findings suggest that lithium thera- py could play a protective role for patients with BD. The additional therapeutic role of vitamin-D in a subgroup of patients with BD could provide a safe, low cost treatment, as well as providing additional advantages for general bone health. However, prospective longitudinal studies are required to understand the pathophysiological processes involved in these processes in BD.

\section{References}

1. Brambilla P, Glahn DC, Balestrieri M, Soares JC. Magnetic resonance findings in bipolar disorder. Psychiatr Clin North Am 2005; 28(2): 443-467.

2. Yoshita M, Fletcher E, DeCarli C. Current concepts of analysis of cerebral white matter hyperintensities on magnetic resonance imaging. Top Magn Reson Imaging 2005; 16(6): 399-407.

3. Moore PB, Shepherd DJ, Eccleston D, Macmillan IC, Goswami U, McAllister VL, et al. Cerebral white matter lesions in bipolar affective disorder: relationship to outcome. Br J Psychiatry 2001; 178: 172-176.

4. Dupont RM, Jernigan TL, Butters N, Delis D, Hesselink JR, Heindel W, et al. Subcortical abnormalities detected in bipolar affective disorder using magnetic resonance imaging. Clinical and neuropsychological significance. Arch Gen Psychiatry 1990; 47: 55-59.

5. Tsai SY, Lee CH, Kuo CJ, Chen CC. A retrospective analysis of risk and protective factors for natural death in bipolar disorder. J Clin Psychiatry 2005; 66(12): 1586-1591.

6. Coffey CE, Figiel GS, Djang WT, Weiner RD. Subcortical hyperintensity on magnetic resonance imaging: a comparison of normal and depressed elderly subjects. Am J Psychiatry 1990; 147: 187-189.

7. Young RC, Biggs JT, Ziegler VE, Meyer DA. A rating scale for mania: reliability, validity and sensitivity. Br J Psychiatry 1978; 133: 429-435.

8. Hamilton M. A rating scale for depression. J Neurol Neurosurg Psychiatry 1960; 23: 56-62.

9. Ware JE, Sherbourne CD. The MOS 36-Item ShortForm Health Survey (SF-36®): I. conceptual framework and item selection. Med Care 1992; 30(6): 473-483.

10. Folstein MF, Folstein SE, McHugh PR. Mini-Mental State: a practical method for grading the cognitive state of patients for the clinician. J Psychiat Res 1975; 12: 189-198. 
11. Huxley N, Baldessarini RJ. Disability and its treatment in bipolar disorder patients. Bipolar Disord. 2007; 9: 183-196.

12. Rosa AR, Franco C, Martinez-Aran A, SanchezMoreno J, Reinares M, Salamero M, et al. Functional impairment in patients with remitted bipolar disorder. Psychother Psychosom 2008; 77: 390-392.

13. Delbello MP, Hanseman D, Adler CM, Fleck DE, Strakowski SM. Twelve-month outcome of adolescents with bipolar disorder following first hospitalization for a manic or mixed episode. Am J Psychiatry 2007; 164: 582-590.

14. Rosa AR, Reinares M, Franco C, Comes M, Torrent C, Sanchez-Moreno J, et al. Clinical Predictors of Functional outcome of bipolar patients in remission. Bipolar Disord 2009; 11: 401-409.

15. Eyles DW, Smith S, Kinobe R, Hewison M, McGrath JJ. Distribution of the vitamin D receptor and 1 alpha-hydroxylase in human brain. J Chem Neuroanat 2005; 29(1): 21-30

16. Berk M, Sanders KM, Pasco JA, Jacka FN, Williams LJ, Hayles AL, et al. Vitamin D deficiency may play a role in depression. Med Hypotheses 2007; 69(6): 1316-1319.

17. Cherniack EP, Troen BR, Florez HJ, Roos BA, Levis S. Some new food for thought: the role of vitamin D in the mental health of older adults. Curr Psychiatry Rep 2009; 11(1): 12-19.

18. Langub MC, Herman JP, Malluche HH, Koszewski NJ. Evidence of functional vitamin D receptors in rat hippocampus. Neuroscience 2001; 104(1): 49-56.
19. Omata N, Murata T, Takamatsu S, Maruoka N, Mitsuya H, Yonekura Y, et al. Neuroprotective effect of chronic lithium treatment against hypoxia in specific brain regions with upregulation of cAMP response element binding protein and brain-derived neurotrophic factor but not nerve growth factor: comparison with acute lithium treatment. Bipolar Disord 2008; 10(3): 360-368.

20. Chuang DM. Neuroprotective and neurotrophic actions of the mood stabilizer lithium: can it be used to treat neurodegenerative diseases? Crit Rev Neurobiol 2004; 16(1-2): 83-90.

21. Camins A, Verdaguer E, Junyent F, Yeste-Velasco M, Pelegrí C, Vilaplana J, et al. Potential mechanisms involved in the prevention of neurodegenerative diseases by lithium. CNS Neurosci Ther 2009; 15(4):333-344.

22. Lyoo IK, Dager SR, Kim JE, Yoon SJ, Friedman SD, Dunner DL, et al. Lithium-induced gray matter volume increase as a neural correlate of treatment response in bipolar disorder: a longitudinal brain imaging study. Neuropsychopharmacology 2010; 35(8): 1743-1750.

Address for correspondence:

Gianluca Serafini, MD

Department of Neuroscience Mental Health and Sensory Functions, "Sapienza" University of Rome, Sant' Andrea Hospital Via Grottarossa 1035-1039, 00189, Rome, Italy Tel. +39-0680345280

Fax +39-0680345342

E-mail: gianluca.serafini@uniroma1.it 